\title{
Erratum: Gestures in Sign Language: Animation and Generation in Real-Time
}

\author{
Nour Ben Yahia and Mohamed Jemni \\ University of Tunis Research Laboratory on Technologies of Information \\ and Communication \& Electrical Engineering (Latice), Tunis, Tunisia \\ benyahia.nour@gmail.com, \\ mohamed.jemniafst.rnu.tn \\ http: //www. latice.rnu.tn
}

K. Miesenberger et al. (Eds.): ICCHP 2014, Part II, LNCS 8548, pp. 482-489, 2014.

(C) Springer International Publishing Switzerland 2014

\section{DOI 10.1007/978-3-319-08599-9_90}

The paper starting on page 482 of this publication has been retracted because a significant amount of material was taken verbatim and without permission from the publication "FABRIK: A fast, iterative solver for the Inverse Kinematics problem" by Andreas Aristidou and Joan Lasenby, (C) 2011 Elsevier Inc. 